\title{
LOWER BOUNDS FOR EIGENVALUES OF STURM-LIOUVILLE PROBLEMS WITH DISCONTINUOUS COEFFICIENTS: INTEGRAL EQUATION METHODS*
}

\author{
BY \\ C. O. HORGAN, J. P. SPENCE AND A. N. ANDRY \\ Michigan State University
}

\begin{abstract}
This paper is concerned with application of the theory of Fredholm integral equations to Sturm-Liouville problems with discontinuous coefficients. Such problems occur naturally in many areas of application involving mechanics of heterogeneous media. Due to the nonsmoothness of the coefficients, the eigenvalue spectrum may exhibit severe irregularities. Lower bounds for eigenvalues are obtained which reflect this behavior. Numerical results are presented for example problems previously treated using other methods.
\end{abstract}

1. Introduction. Eigenvalue problems with discontinuous coefficients have been the subject of much recent attention. The widespread interest in such problems stems from their natural appearance in diverse fields of application. For example, the propagation of harmonic waves in elastic composites with periodic structure gives rise to Sturm-Liouville problems with discontinuous coefficients subject to quasi-periodic boundary conditions (see e.g. [1-3] and the references cited therein). Also, vibration problems arising in geophysics lead to consideration of Sturm-Liouville problems with discontinuous coefficients $[4,5]$. Other examples include heat conduction in layered media [6-8] and vibration problems in structural mechanics [9].

Considerable emphasis has been placed on the development of computational schemes for estimating eigenvalues and eigenfunctions for such problems. These efforts have met with serious difficulties due to nonsmoothness of the coefficients and the resulting spectral irregularities. Early attempts [1,2] were focused mainly on variational techniques (e.g., Rayleigh-Ritz approximation and mixed variational schemes) with emphasis on obtaining upper bounds for eigenvalues. Alternative methods, such as finite difference and finite element methods, leading to matrix eigenvalue problems have been investigated $[10,11]$, as well as direct variational schemes [12]. More recently, results from classical SturmLiouville theory and eigenvalue optimization techniques have been adapted for these problems $[3,7,8]$ to obtain upper and lower bounds for eigenvalues. Lower bounds using Weinstein's method of intermediate problems have also been established [13].

It is the purpose of this paper to present an integral equation approach for investigation of eigenvalue problems with discontinuous coefficients, with emphasis on obtaining lower bounds $^{1}$ for eigenvalues. We confine our attention to the simplest prototype Sturm-

* Received September 4, 1980. This work was supported in part by the Division of Engineering Research, Michigan State University.

${ }^{1}$ The methods used in [6-8], [12] are satisfactory in obtaining accurate upper bounds. 
Liouville problem (Eqs. (1), (2), Sec. 2) arising in heat conduction in a layered composite. This problem is first converted to Liouville normal form (Eqs. (5), (6)). In Sec. 3, using a standard technique involving a Green's function, we transform this problem to a Fredholm integral equation of the second kind. The kernel, though discontinuous, is symmetric and square-integrable. Standard results from integral equation theory are then adapted in Sec. 4 to provide lower bounds for eigenvalues. A direct lower bound is found for the smallest eigenvalue (Eq. (20)), while lower bounds are obtained for higher eigenvalues (Eq. (21)) by employing a set of upper bounds from other methods. Alternative lower bounds [8] based on eigenvalue optimization techniques are briefly discussed. Illustrative examples are described in Sec. 5, with numerical results presented in Sec. 6. The results suggest that integral operator techniques, with inherent "smoothing" properties, may be particularly appropriate for investigation of the problems of concern here. ${ }^{2}$

2. Sturm-Liouville problems with discontinuous coefficients. We consider the eigenvalue problem

$$
\begin{gathered}
\left(\kappa u^{\prime}\right)^{\prime}+\lambda c u=0, \quad 0<x<1 \\
u(0)=0, \quad u(1)=0,
\end{gathered}
$$

where $\kappa(x), c(x)$ are positive functions, bounded on $[0,1]$. We assume that the coefficients $\kappa(x), c(x)$ have step discontinuities at a finite set of points $x_{1}, \ldots, x_{n}$ on $(0,1)$, are continuous elsewhere and are such that the eigenvalue problem (1), (2) admits an infinite set of distinct eigenvalues $0<\lambda_{1}<\lambda_{2} \ldots$. In the context of heat conduction in layered composites, for example, $\kappa(x)$ is the heat conductivity and $c(x)$ the heat capacity, both subject to possibly large discontinuities at material interfaces.

A complete spectral theory for Sturm-Liouville problems with discontinuous coefficients has not yet been established. Several phenomena not found in the classical case have been observed, however. The asymptotic behavior of the eigenvalues has been shown to have a "solotone" effect [5], a result of major significance for geophysical inverse problems. The eigenvalue spectrum may exhibit severe irregularities, depending on the coefficients (see the numerical results in Sec. 6 here). Many results from the continuous case do carry over formally to the discontinuous case, but these may be difficult (or inefficient) in implementation. An example of this concerns the classical Rayleigh-Ritz procedure for obtaining upper bounds on eigenvalues. It has been shown that a direct application of this approach to the problem (1), (2) in general yields poor results, particularly when the coefficients have large discontinuities. An alternative scheme, based on mixed variational principles and leading to a modified Rayleigh quotient ("new quotient"), has been developed (see e.g. [2] and the references cited therein). The underlying numerical analysis, with rigorous convergence estimates, has been discussed in [15].

It has been demonstrated recently [7] that conversion of the problem (1), (2) to Liouville normal form leads to computational advantages. Thus, we let

$$
\begin{aligned}
T=\int_{0}^{1} \kappa^{-1}(s) d s, \quad t & =T^{-1} \int_{0}^{x} \kappa^{-1}(s) d s, \quad v(t)=u(x(t)), \\
f(t) & =T^{2} \kappa(x(t)) c(x(t)) .
\end{aligned}
$$

\footnotetext{
${ }^{2}$ We remark that integral equation techniques have proved to be particularly successful in analysis of eigenvalue problems with random coefficients [14].
} 
Then the eigenvalues $\lambda_{i}(i=1,2, \ldots)$ are the eigenvalues of

$$
\ddot{v}+\lambda f v=0, \quad 0<t<1, \quad v(0)=0, \quad v(1)=0,
$$

where the superposed dot represents differentiation with respect to $t$. The coefficient $f(t)$ is positive and bounded on $[0,1]$ and has discontinuities at the points $t_{i}=T^{-1} \int_{0}^{x_{i}} \kappa^{-1}(s) d s$. The effect of the foregoing transformation has been to remove the discontinuous coefficient $\kappa$ from its position subject to differentiation in (1). It is shown in [7] that application of the Rayleigh-Ritz technique to the transformed problem (5), (6) leads to accurate upper bounds for $\lambda_{1}$ with minimal computational effort. Explicit lower bounds for $\lambda_{1}$ in terms of the coefficients have also been obtained in [7], [8]. Our primary purpose in this paper is to provide an integral equation formulation for the basic problem (1), (2) (or equivalently (5), (6)) and thereby develop alternative lower-bound estimates, particularly for higher eigenvalues for which few results are known.

3. Integral equation formulation. The eigenvalue problem (5), (6) is readily transformed to the integral equation

$$
v(t)=\lambda \int_{0}^{1} G(t, s) f(s) v(s) d s,
$$

where

$$
G(t, s)=(1-s) t, \quad 0 \leq t \leq s ; \quad(1-t) s, \quad s \leq t \leq 1
$$

is the Green's function for the problem

$$
-\ddot{G}=0, \quad G(0)=0, \quad G(1)=0 .
$$

Note that if we view the kernel of the integral equation as

$$
\tilde{K}(t, s)=G(t, s) f(s)
$$

then the kernel is not symmetric. However, we may obtain an integral equation with a symmetric kernel by multiplying (7) by $\sqrt{f(t)}$ and so obtaining

$$
\sqrt{f(t)} v(t)=\lambda \int_{0}^{1} \sqrt{f(t)} G(t, s) \sqrt{f(s)} \sqrt{f(s)} v(s) d s .
$$

We may write (11) in the form

$$
\psi(t)=\lambda \int_{0}^{1} K(t, s) \psi(s) d s
$$

where

$$
\psi(t)=\sqrt{f(t)} v(t), \quad K(t, s)=\sqrt{f(t)} G(t, s) \sqrt{f(s)} .
$$

Eq. (12) is a Fredholm integral equation of the second kind. It may be readily verified that the kernel $K(t, s)$, even though discontinuous, is real, symmetric, square-integrable and continuous in the mean ([16], p. 502).

For the integral Eq. (12) the iterated kernels $K_{n}$ are defined by:

$$
K_{1}=K ; \quad K_{n+1}(t, s)=\int_{0}^{1} K(t, z) K_{n}(z, s) d z .
$$


The trace and $L^{2}$ norm of $K$ are

$$
\begin{gathered}
\operatorname{tr}(K)=\int_{0}^{1} K(t, t) d t \\
\|K\|=\left(\int_{0}^{1} \int_{0}^{1}|K(t, s)|^{2} d t d s\right)^{1 / 2}
\end{gathered}
$$

For symmetric $K,(15)-(17)$ give

$$
\|K\|^{2}=\operatorname{tr}\left(K_{2}\right)
$$

If $0<\lambda_{1}<\lambda_{2}, \ldots$, is the complete sequence of eigenvalues of (12), then [17]

$$
\operatorname{tr}\left(K_{n}\right)=\sum_{m=1}^{\infty}\left(\frac{1}{\lambda_{m}}\right)^{n}, \quad n \geq 2
$$

4. Lower bounds for eigenvalues. A lower bound for $\lambda_{1}$ follows directly from (18), (19):

$$
\lambda_{1} \geq(\|K\|)^{-1} \text {. }
$$

If $\left\{\bar{\lambda}_{i}\right\}_{i=1}^{N}$ is any set of upper bounds to the least $N$ eigenvalues, (18), (19) give

$$
\lambda_{M} \geq\left(\|K\|^{2}-\sum_{\substack{i=1 \\ i \neq M}}^{N}\left(\bar{\lambda}_{i}\right)^{-2}\right)^{-1 / 2}, \quad M=1,2, \ldots, N+1 .
$$

In the next two sections, we consider some particular example problems and provide numerical results to assess the accuracy of the bounds (20), (21).

Alternative lower bounds have been obtained in [7], [8] based on the Liouville normal form (5), (6) and using results of Krein [18] on eigenvalue extremization. In [18], Krein is concerned with the problem of maximizing and minimizing the eigenfrequencies $\lambda_{n}(f)$ of a string of variable density $f$ (i.e., problem (5), (6)) subject to the constraints on $f$ of a fixed total mass

$$
\int_{0}^{1} f(t) d t=M
$$

and various bounds on the density, such as

$$
0<f \leq H(H \geq M) \text { or } 0<h \leq f(h \leq M) .
$$

Under conditions (22), $(23)_{2}$ the minimum $\lambda_{1}$ is attained by the singular function

$$
f(t)=h+(M-h) \delta\left(t-\frac{1}{2}\right),
$$

which may be viewed as a uniform string with a bead at the center. Under conditions (22), (23) $)_{1}$ the minimum $\lambda_{1}$ is attained by

$$
\begin{aligned}
f(t) & =H, & & \left|t-\frac{1}{2}\right|<\frac{1}{2} \frac{M}{H}, \\
& =0 & & \text { otherwise. }
\end{aligned}
$$

The minimizing functions for the higher eigenvalues $\lambda_{n}$ have $n$ equally spaced beads or dense intervals placed at the antinodal points of the $n$th eigenfunction of a uniform string 
[18]. Thus we see that Krein's work [18] is directly related to investigation of eigenvalue problems with discontinuous coefficients and this relationship was exploited in [7], [8].

Under the conditions (22), (23) $)_{1}$, Krein [18] has shown that

$$
\lambda_{n}(f) \geq \frac{4 n^{2} H}{M^{2}} \chi\left(\frac{M}{H}\right)
$$

and under $(22),(23)_{2}$,

$$
\lambda_{n}(f) \geq \frac{4 n^{2}}{h} \chi\left(\frac{h}{M}\right)
$$

where $\chi(d)$ is the least positive root of the transcendental equation

$$
\sqrt{ } \chi \tan \sqrt{ } \chi=d / 1-d
$$

The lower bounds (26), (27) may be made explicit on obtaining bounds for the lowest root of (28). Thus Krein shows that

$$
\chi(d)>d\left[1-\frac{4 d}{3}+\left(\frac{11}{24}+\frac{4}{\pi^{4}}\right) d^{2}\right]^{-1 / 2} .
$$

This result, in conjunction with (26), was utilized in [8] for $n=1$, and will be further considered in Secs. 5, 6 here.

5. Illustrative examples. To assess the accuracy of the lower bounds discussed in Sec. 4 we now consider some specific examples. The case of heat conduction through a composite composed of two identical homogeneous outer layers enclosing an inner homogeneous layer has been treated in [6-8]. Thus the coefficients $k(x), c(x)$ in (1) are piecewise constant and are given by

$$
\begin{gathered}
c_{1}, \kappa_{1} \quad \text { in } 0 \leq x<(1-b) / 2, \quad(1+b) / 2<x \leq 1, \\
c_{2}, \kappa_{2} \text { in }(1-b) / 2 \leq x \leq(1+b) / 2 .
\end{gathered}
$$

The eigenvalue problem (1), (2) with coefficients given by (30), (31) has been solved exactly in [6]. Solutions to Eq. (1) were obtained in each region, satisfying the boundary conditions (2) and then matched by ensuring continuity of $u$ and $\kappa u^{\prime}$ at the interfaces. In this way, transcendental equations governing the exact eigenvalues were found and solved numerically for various combinations of the constants $c_{\beta}, \kappa_{\beta}, \beta=1,2$.

It is convenient to introduce the following notation:

$$
\begin{aligned}
& \gamma=\kappa_{2} / \kappa_{1}, \quad \theta=c_{2} / c_{1}, \quad n_{1}=1-b, \quad n_{2}=b, \\
& \bar{\kappa}=n_{1} \kappa_{1}+n_{2} \kappa_{2}, \quad \bar{c}=n_{1} c_{1}+n_{2} c_{2},
\end{aligned}
$$

and so, using the normalization $\bar{\kappa}=1, \bar{c}=1$, we obtain

$$
\begin{aligned}
& c_{1}=\left(n_{1}+n_{2} \theta\right)^{-1}, \quad c_{2}=\theta\left(n_{1}+n_{2} \theta\right)^{-1} ; \quad \kappa_{1}=\left(n_{1}+n_{2} \gamma\right)^{-1}, \\
& \kappa_{2}=\gamma\left(n_{1}+n_{2} \gamma\right)^{-1} .
\end{aligned}
$$

The corresponding dimensionless eigenvalue is then denoted by $v$ and given by

$$
v \equiv(\lambda \bar{c} / \bar{\kappa})^{1 / 2}=\lambda^{1 / 2} .
$$


For given values of the geometric parameters $n_{1}, n_{2}$, the effect of the material discontinuities on $v$ is conveniently analyzed through consideration of the dependence of $v$ on the dimensionless material parameters $\gamma$ and $\theta$. For continuous conductivities $\gamma=1$ while for continuous capacities $\theta=1$. Henceforth, we will set $b=\frac{1}{2}\left(n_{1}=n_{2}=\frac{1}{2}\right)$ and so the jumps in $\kappa, c$ occur at $x_{1}=\frac{1}{4}$ and $x_{2}=\frac{3}{4}$. The transcendental equations for the exact eigenvalues $v$ referred to above are then given by

$$
\begin{aligned}
& \sqrt{\gamma \theta} \sin \beta v \sin \alpha v-\cos \beta v \cos \alpha v=0, \\
& \sqrt{\gamma \theta} \cos \beta v \sin \alpha v+\sin \beta v \cos \alpha v=0,
\end{aligned}
$$

where

$$
\alpha=\frac{1}{4}\left(\frac{1+\gamma}{1+\theta}\right)^{1 / 2}, \quad \beta=\frac{1}{4}\left(\frac{1+\gamma^{-1}}{1+\theta^{-1}}\right)^{1 / 2} .
$$

The coefficient $f(t)$ in Eq. (5) is given by

$$
\begin{array}{rlrl}
f(t) & =h & , & \left|t-\frac{1}{2}\right|>\frac{1}{2(\gamma+1)} \\
& =H \quad, \quad & \left|t-\frac{1}{2}\right|<\frac{1}{2(\gamma+1)}
\end{array}
$$

where

$$
h=\frac{(\gamma+1)^{3}}{4 \gamma^{2}(1+\theta)}, \quad H=\gamma \theta h .
$$

The kernel norm (18) for the integral Eq. (12) then becomes

$$
\|K\|^{2}=h^{2}\left\{\frac{1}{90}-\frac{(H / h-1)^{2}}{144} l(1-l)^{3}\left(3+l^{2}\right)+\frac{(H / h)^{2}-1}{720} l\left(15-10 l^{2}+3 l^{4}\right)\right\},
$$

where

$$
l=1 /(1+\gamma) \text {. }
$$

Substitution of (39), (40) in (20) yields an explicit lower bound for the dimensionless eigenvalue $v_{1}(\gamma, \theta)$ as

$$
v_{1} \geq(\|K\|)^{-1 / 2}
$$

where $\|K\|$ is given by (39).

When $f$ is given by (37), we find that $M$ as defined in (22) is

$$
M=(\gamma+1)^{2} / 4 \gamma .
$$

Thus, from (26), (27) (on using (29)) we obtain, for $\gamma \theta \geq 1$,

$$
v_{n} \geq \frac{4 n \gamma^{1 / 2}}{\gamma+1}\left[1-\frac{4}{3}\left(\frac{1+\theta^{-1}}{1+\gamma}\right)+\left(\frac{11}{24}+\frac{4}{\pi^{4}}\right)\left(\frac{1+\theta^{-1}}{1+\gamma}\right)^{2}\right]^{-1 / 4},
$$

and

$$
v_{n} \geq \frac{4 n \gamma^{1 / 2}}{\gamma+1}\left[1-\frac{4}{3}\left(\frac{1+\gamma^{-1}}{1+\theta}\right)+\left(\frac{11}{24}+\frac{4}{\pi^{4}}\right)\left(\frac{1+\gamma^{-1}}{1+\theta}\right)^{2}\right]^{-1 / 4},
$$


TABLE 1. Comparison of lower bounds $\underline{v}_{1}$ for the least eigenvalue $v_{1}\left(\%\right.$ R.E. $\left.=100\left(v_{1}-\underline{v}_{1}\right) / v_{1}\right)$.

\begin{tabular}{|c|c|c|c|c|}
\hline$\theta$ & 0.1 & 1 & 10 & 100 \\
\hline $\begin{array}{c}\text { Exact } v_{1} \\
0.1(43)(\% \text { R.E. }) \\
(41)(\% \text { R.E. })\end{array}$ & $\begin{array}{l}5.05808 \\
1.19009(76.4) \\
4.44725(12.1)\end{array}$ & $\begin{array}{l}2.42132 \\
1.43035(40.9) \\
2.36393(2.38)\end{array}$ & $\begin{array}{l}1.80629 \\
1.80135(.273) \\
1.77092(1.96)\end{array}$ & $\begin{array}{l}1.73177 \\
1.72653(.303) \\
1.69822(1.94)\end{array}$ \\
\hline 1 & $\begin{array}{l}4.21128 \\
2.48774(40.9) \\
3.94741(6.27)\end{array}$ & $\begin{array}{l}3.14159 \\
3.13301(.273) \\
3.08007(1.96)\end{array}$ & $\begin{array}{l}2.52922 \\
2.48774(1.64) \\
2.50428(.986)\end{array}$ & $\begin{array}{l}2.44333 \\
2.43646(.281) \\
2.42059(.931)\end{array}$ \\
\hline 10 & $\begin{array}{l}1.80629 \\
1.80135(.273) \\
1.77092(1.96)\end{array}$ & $\begin{array}{l}1.45420 \\
1.43035(1.64) \\
1.44876(.374)\end{array}$ & $\begin{array}{l}1.22511 \\
1.19009(2.86) \\
1.22457(.044)\end{array}$ & $\begin{array}{l}1.19037 \\
1.18666(.312) \\
1.18994(.036)\end{array}$ \\
\hline 100 & $\begin{array}{l}0.596433 \\
0.594627(.303) \\
0.587457(1.51)\end{array}$ & $\begin{array}{l}0.483827 \\
0.482468(.281) \\
0.482532(.268)\end{array}$ & $\begin{array}{l}0.409971 \\
0.408694(.312) \\
0.409948(.006)\end{array}$ & $\begin{array}{l}0.398675 \\
0.397366(.328) \\
0.398673(<.001)\end{array}$ \\
\hline
\end{tabular}

respectively. When $\theta>\gamma,(43)_{1}$ provides a sharper lower bound than $(43)_{2}$ and conversely. For the case $n=1$, the bounds (43) 1,2 have been previously obtained in [8] (see Eqs. (6.12) $)_{1,2}$ in [8]). When $\gamma \theta \leq 1$, from (38) it can be seen that the roles of $H$ and $h$ should be interchanged in (26), (27). Thus we obtain

$$
v_{n} \geq \frac{4 n \gamma^{1 / 2}}{\gamma+1}\left[1-\frac{4}{3}\left(\frac{1+\theta}{1+\gamma^{-1}}\right)+\left(\frac{11}{24}+\frac{4}{\pi^{4}}\right)\left(\frac{1+\theta}{1+\gamma^{-1}}\right)^{2}\right]^{-1 / 4},
$$

and

$$
v_{n} \geq \frac{4 n \gamma^{1 / 2}}{\gamma+1}\left[1-\frac{4}{3}\left(\frac{1+\gamma}{1+\theta^{-1}}\right)+\left(\frac{11}{24}+\frac{4}{\pi^{4}}\right)\left(\frac{1+\gamma}{1+\theta^{-1}}\right)^{2}\right]^{-1 / 4} .
$$

6. Numerical results and discussion. To assess the accuracy and nature of the error involved in the lower bounds (20) and (21), a number of bounds were computed for various combinations of material properties. The lower bound (41) for the least eigenvalue from the integral equation is compared to the lower bound (43) (when $n=1)$ and the actual ${ }^{3}$ least eigenvalue in Table 1. The lower bounds (21) for higher eigenvalues are obtained using upper bounds from Galerkin's method with trigonometric test functions applied to the differential equation (5). The results for five-term eigenfunction approximations are shown in Table 2. The best possible lower bounds for higher eigenvalues obtainable from (21) for a given number of upper bounds are presented in Table 3. It should be noted that the bounds (43) for higher eigenvalues do not reflect irregularities in the spectrum and so will not be considered further in this paper.

${ }^{3}$ All the exact eigenvalues presented in this paper are computed from the transcendental equations (35). 
Table 1 provides a comparison between th $\downarrow$ lower bound (41) for the least eigenvalue obtained from the integral equation and the bounds (43) using constrained eigenvalue minimization methods [8]. In all cases where the Liouville normal form (5) has a centerweighted density $f(t)$, that is, when the product of the discontinuity ratios, $\gamma \theta$, exceeds or equals one, the bounds based on (43) are quite accurate. When both ratios $\gamma$ and $\theta$ exceed one, ${ }^{4}$ the bounds based on (41) compare favorably with those from (43). In mixed cases where either $\gamma$ or $\theta$, but not both, and the product $\gamma \theta$ are greater than or equal to one, the bounds (43) are somewhat better than (41). However, in the edge-weighted cases where the product $\gamma \theta$ is less than one, the coefficient $f(t)$ in (37) is no longer similar to the extremizing density (25) of Krein, and the bounds (43) are quite poor. In these cases, the accuracy of (41) is also less than when $\gamma \theta$ exceeds one but for quite a different reason.

The error in the bound (20) (and thus (41)) depends on the appropriateness of truncation of the series (19) which adds to $\|K\|^{2}$, and thus depends on the distribution of the spectrum. The closer the least eigenvalue is to the rest of the spectrum, the less accurate the bound (41) can be. Table 1 shows that the accuracy of (41) is exceptional for highly center-weighted cases $(\gamma \theta \gg 1)$ and falls off for the edge-weighted cases $(\gamma \theta<1)$. The string analogy allows an interpretation of this phenomenon. In the center-weighted cases, the concentration of the mass in the center near the antinodal point of the first mode significantly lowers the fundamental frequency compared to that of a uniform string of the lower density near the ends. However, the mass at the nodal point of the second mode affects the second frequency of the light uniform string much less. Thus the first two frequencies are well separated (see e.g., Table 2, Case 3). In contrast, in the edge-weighted cases where $\gamma \theta$ is less than 1 , the motion of the concentrated mass at the ends is comparable in the first and second mode, and the frequencies are correspondingly closer than those of the uniform string (see e.g., Table 2, Case 4). Such clustering of the lower eigenvalues decreases the accuracy of the bound (20) (i.e. (41)).

The use of upper bounds for the eigenvalues in (21) can remedy this defect in (20) to some extent and also supply lower bounds for higher eigenvalues. In Table 2, upper bounds are obtained by applying Galerkin's method, with eigenfunction approximations of the form

$$
v_{i}=\sum_{j=1}^{5} c_{i j} \sin j \pi t,
$$

to the differential equation (5). (Alternatively, upper bounds may be found using the methods of [6-8], [12]. Of course, integral equation methods may also prove particularly effective in obtaining upper bounds, but we do not pursue this in the present paper.) These five upper bounds are then used in (21) to obtain lower bounds for the first six eigenvalues, as shown in Table 2.

The error in the lower bound (21) comes from two sources: truncation of the series and approximation of the second (correction) term in (21). The error due to series truncation depends on the number of terms used in the correction term as well as the distribution of the spectrum. The error due to approximation of the correction term depends primarily on the accuracy of the upper bound for the least eigenvalue used in the correction term. In Table 2, Case 1, the eigenvalues are nearly equally spaced and the upper bounds are quite accurate. In this case, the error in (21) is primarily due to truncation after the fifth term of

${ }^{4}$ We remark that these are the only cases considered in [8]. 
TABLE 2. Lower bounds (21) for higher eigenvalues based on upper bounds from Galerkin's method.

\begin{tabular}{|c|c|c|c|c|c|c|}
\hline Case 1: $\gamma=0.1$ & $\theta=100$ & & & & & \\
\hline$i$ & 1 & 2 & 3 & 4 & 5 & 6 \\
\hline Upper bound & 1.7318 & 3.4692 & 5.2174 & 6.9821 & 8.7667 & \\
\hline Exact $v_{i}$ & 1.7318 & 3.4691 & 5.2164 & 6.9767 & 8.7514 & 10.540 \\
\hline Lower bound (21) $\underline{v}_{i}$ & 1.7310 & 3.4436 & 5.0343 & 6.3168 & 7.1786 & 8.3342 \\
\hline \% R.E. & 0.05 & 0.73 & 3.5 & 9.5 & 18 & 21 \\
\hline Case $2: \gamma=100$ & $\theta=0.1$ & & & & & \\
\hline Upper bound & 0.59697 & 1.2984 & 1.8096 & 2.5966 & 3.0676 & \\
\hline Exact $v_{i}$ & 0.59643 & 1.2984 & 1.7966 & 2.5966 & 3.0140 & 3.8493 \\
\hline Lower bound (21) $\underline{v}_{i}$ & 0.59614 & 1.2605 & 1.6420 & 1.9705 & 2.0592 & 2.1794 \\
\hline \% R.E. & 0.05 & 2.9 & 8.6 & 24 & 32 & 43 \\
\hline Case 3: $\gamma=10$, & $\theta=10$ & & & & & \\
\hline Upper bound & 1.2388 & 8.7532 & 12.520 & 16.377 & 24.634 & \\
\hline Exact $\quad v_{i}$ & 1.2251 & 6.2832 & 11.341 & 12.566 & 13.791 & 18.850 \\
\hline Lower bound (21) $\underline{v}_{i}$ & 1.2247 & 2.6561 & 2.6604 & 2.6612 & 2.6616 & 2.6617 \\
\hline$\%$ R.E. & 0.03 & 58 & 77 & 79 & 81 & 86 \\
\hline Case 4: $\gamma=0.1$, & $\theta=0.1$ & & & & & \\
\hline Upper bound & 5.3157 & 8.7533 & 10.827 & 16.377 & 21.840 & \\
\hline Exact $v_{i}$ & 5.0581 & 6.2832 & 7.5083 & 12.566 & 17.624 & 18.850 \\
\hline Lower bound (21) $\underline{v}_{i}$ & 4.5688 & 5.3585 & 5.4720 & 5.5467 & 5.5592 & 5.5651 \\
\hline$\%$ R.E. & 9.7 & 15 & 27 & 56 & 68 & 70 \\
\hline
\end{tabular}

the series, and the accuracy of the lower bounds is quite good. In Case 2, the spectral distribution is similar to Case 1, but the upper bound to the least eigenvalue is less accurate. The errors in the lower bounds are correspondingly greater, excepting the lower bound for the least eigenvalue, since it is independent of the less accurate upper bound to $v_{1}$. In Cases 3 and 4 , there is significant irregularity in eigenvalue spacing and the upper bounds are much less accurate than in Cases 1 and 2. The accuracies of lower bounds to corresponding eigenvalues are quite different in Cases 3 and 4, however. Within each case, the accuracies of lower bounds within a cluster of closely spaced eigenvalues are comparable. In Case 3 , the lower bound to the isolated least eigenvalue is accurate, and the lower bounds to the cluster $v_{3}-v_{5}$ are of $77-81 \%$ relative error. In Case 4 , one cluster is $v_{1}-v_{3}$ and the corresponding lower bounds are of $9.7-27 \%$ relative error, $v_{4}$ is isolated with $56 \%$ relative error in the lower bound, and $v_{5}-v_{7}\left(v_{7}\right.$ is not tabulated $)$ is another cluster with $68-72 \%$ relative error in the lower bounds.

Table 3 presents another examination of Case 4 designed to isolate the effects of the number of bounds used and of the spectral distribution from that of the accuracy of the upper bounds used. Thus in Table 3, the actual eigenvalues are used as upper bounds in (21) 
TABLE 3. Best possible lower bounds (21) for $N$ upper bounds. Case $4: \gamma=0.1, \theta=0.1$.

\begin{tabular}{|l|c|c|c|c|c|c|}
\hline \multicolumn{1}{|c|}{$\begin{array}{c}\text { Exact } v_{i} \\
\text { En }\end{array}$} & 1 & 2 & 3 & 4 & 5 & 6 \\
\hline$\underline{v}_{i}(20)$ & 4.0580758 & 6.2831853 & 7.5082948 & 12.566371 & 17.624447 & 18.849556 \\
$\%$ R.E. & $(12)$ & & & & & \\
& & & & & \\
$\underline{v}_{i}(21 ; N=1)$ & 4.4472526 & 5.5838400 & & & & \\
$\%$ R.E. & $(12)$ & $(11)$ & & & & \\
$N=2$ & 4.7804542 & 5.5838400 & 7.1295907 & & & \\
& $(5.5)$ & $(11)$ & $(5)$ & & & \\
$N=3$ & 4.9998870 & 6.1175290 & 7.1295907 & 10.841940 & & \\
& $(1.1)$ & $(2.6)$ & $(5)$ & $(14)$ & & \\
$N=4$ & 5.0317128 & 6.2065749 & 7.3272473 & 10.841940 & 13.267766 & \\
& $(0.52)$ & $(1.3)$ & $(2.4)$ & $(14)$ & $(25)$ & \\
$N=5$ & 5.0401048 & 6.2306707 & 7.3830171 & 11.269071 & 13.267766 & 14.616953 \\
& $(0.36)$ & $(0.84)$ & $(1.7)$ & $(10)$ & $(25)$ & $(22)$ \\
\hline
\end{tabular}

to obtain sequences of lower bounds. Again, within a cluster of closely spaced eigenvalues, the error is seen to be comparable, for a given number of upper bounds. One obvious effect of the number of upper bounds used is the increase in accuracy of the lower bounds. A more subtle effect is the interaction with the effects of clustering. When upper bounds for all members of a cluster are used, there is a significant improvement in the accuracy of the lower bounds in that cluster which is greater than the improvement obtained when adding a bound to an isolated eigenvalue. For example, examine the relative error changes down the columns in Table 3. When the third upper bound is added, the relative error in columns 1 and 2 drops sharply; the addition of the fourth bound cuts the error by a more typical rate.

One other source of error in these series approximations which must be mentioned is that of the numerical truncation errors inherent in summing numbers of disparate magnitudes and in taking smaller differences of larger numbers. Both problems occur in using (21) to bound the higher eigenvalues. Any use of this method must take account of these sources of error by summing from small to large and using as many digits as possible.

\section{REFERENCES}

[1] E. H. Lee, A survey of variational methods for elastic wave propagation analysis in composites with periodic structures, in Dynamics of Composites (E. H. Lee, ed.) ASME, New York (1972), 122-138

[2] S. Nemat-Nasser and S. Minagawa, Harmonic waves in layered composites : comparison among several schemes, J. Appl. Mech. 42, 699-704 (1975)

[3] C. O. Horgan, K.-W. Lang and S. Nemat-Nasser, Harmonic waves in layered composites: new bounds on eigenfrequencies, J. Appl. Mech. 45, 829-833 (1978)

[4] R. S. Anderssen and J. R. Cleary, Asymptotic structure in torsional free oscillations of the earth I-overtone structure, Geophys. J. R. Astr. Soc. 39, 241-268 (1974) 
[5] A. McNabb, R. S. Anderssen and E. R. Lapwood, Asymptotic behavior of the eigenvalues of a Sturm-Liouville system with discontinuous coefficients, J. Math. Anal. Appl. 54, 741-751 (1976)

[6] S. Nemat-Nasser and K.-W. Lang, Eigenvalue problems for heat conduction in composite materials, Iranian J. Sci. Technology 7, 243-260 (1979)

[7] C. O. Horgan and S. Nemat-Nasser, Bounds on eigenvalues of Sturm-Liouville problems with discontinuous coefficients. J. of Appl. Math. and Phys. (ZAMP), 30, 77-86 (1979)

[8] C. O. Horgan and S. Nemat-Nasser, Variational methods for eigenvalue problems in composites, Proceedings of the IUTAM Symposium on Variational Methods in the Mechanics of Solids, Northwestern University, Sept. 1978 (S. Nemat-Nasser, ed.), Pergamon Press, New York (1980), 52-58

[9] K.-W. Lang and S. Nemat-Nasser, Vibration and buckling of composite beams, J. Struct. Mech. 5, 395-419 (1977)

[10] E. H. Lee and W. H. Yang, On waves in composite materials with periodic structure, SIAM J. Appl. Math. 25, 492-499 (1973)

[11] G. H. Golub, L. Jenning and W. H. Yang, Waves in periodically structured media, J. of Computational Phys. 17, 349-357 (1975)

[12] D. H. Hodges, Direct solution for Sturm-Liouville systems with discontinuous coefficients, AIAA Journal 17, 924-926 (1979)

[13] W. B. Bickford, Lower bounds to eigenvalues of piecewise continuous elastic systems, J. of Appl. Math. and Phys. (ZAMP), 30, 65-75 (1979)

[14] B. E. Goodwin and W. E. Boyce, The vibrations of a random elastic string : the method of integral equations, Quart. Appl. Math. 22, 261-266(1964)

[15] I. Babuška and J. E. Osborn, Numerical treatment of eigenvalue problems for differential equations with discontinuous coefficients, Math. of Computation 32, 991-1023 (1978)

[16] L. Collatz, The numerical treatment of differential equations, (3rd ed.), Springer-Verlag, Berlin, 1960

[17] J. A. Cochran, Analysis of linear integral equations, McGraw-Hill, New York, 1972

[18] M. G. Krein, On certain problems on the maximum and minimum of characteristic values and on the Lyapunov zones of stability, AMS Translations Ser. 2, 1, 163-187 (1955) 